\title{
Propagación in vitro de Tropaeolum tuberosum, "mashua" de pulpa de color aplicando dosis crecientes del 6-bencilaminopurina
}

\section{In vitro propagation of Tropaeolum tuberosum, "mashua" of colored pulp applying increasing doses of 6- benzylaminopurine}

Segundo Eloy Lopéz- Medina ${ }^{1 *}(\mathbb{D})$, José Mostacero- León ${ }^{1}(\mathbb{D})$, Angélica Lopéz - Zavaleta ${ }^{1}$ (D) Armando Efraín Gil- Rivero ${ }^{1}$ (D), Anthony J. De La Cruz-Castillo' iD

\section{RESUMEN}

Tropaeolum tuberosum "mashua", es una planta herbácea de la familia Tropaeolaceae. Se cultiva en Perú y en Bolivia entre los 3,000 a 4,000 m.s.n.m., pudiéndose encontrar ecotipos de diversos colores, como la mashua negra. Este recurso ocupa el tercer lugar después de oca y el olluco, actualmente es un cultivo de exportación, por sus propiedades nutritivas y culinarias. Siendo necesario la realización de protocolos de conservación in vitro, por su contenido de principios activos. Ante la necesidad de un mayor conocimiento se planteó como objetivo de investigación determinara la concentración más adecuada de la 6-bencilaminopurina en la propagación in vitro de $T$. tuberosum. El material biológico procedió de la estación experimental Carabamba de la Universidad Nacional de Trujillo, se transportaron tubérculos los cuales fueron acondicionados en invernadero. Para la obtención de plantas madres, se colectaron los explantes (nudos) y se llevaron al Laboratorio de Biotecnología del Instituto de la Papa y Cultivos Andinos. Donde fueron sembrados en medio MS (1962), suplementado con $3 \%$ de sacarosa, agar al $0.8 \%$ y Bencilaminopurina (BAP), a la dosis creciente de $0 ; 0.5 ; 1.50$ y $2.5 \mathrm{ppm}$, los cuales constituyeron los tratamientos en un diseño en bloques completos al azar. Se encontró que aplicando dosis crecientes del 6-bencilaminopurina el tratamiento 3 a 1.5 ppm de 6-bencilaminopurina, fue el más satisfactorio en cuanto al número de brotes por explanto. Se concluye que el BAP a 1.50 ppm es la concentración más óptima para la propagación de T. tuberosum "mashua negra".

Palabras clave: Tropaeolum, mashua, in vitro, biotecnología, pulpa de color.

\begin{abstract}
Tropaeolum tuberosum, is an herbaceous plant of the Tropaeolaceae family. It is known as "mashua", both in Peru and in Bolivia. Where it is grown between 3,000 to 4,000 m.a.s.1., it is possible to find ecotypes of various colors, such as black mashua. This resource occupies the third place after oca and olluco, it is currently an export crop, for its nutritional and culinary properties. Being necessary to carry out in vitro conservation protocols, due to its content of active ingredients. In view of the need for greater knowledge, the objective of research was to determine the most adequate concentration of 6-benzylaminopurine in the in vitro propagation of T. tuberosum. The biological material came from the Carabamba experimental station of the National University of Trujillo, tubers were transported which were conditioned in the greenhouse. To obtain mother plants, the explants (nodes) were collected and taken to the Biotechnology Laboratory of the Institute of Potato and Andean Crops. Where they were seeded in MS medium (1962), supplemented with 3\% sucrose, $0.8 \%$ agar and benzylaminopurine (BAP), at the increasing dose of $0 ; 0.5$; 1.50 and $2.5 \mathrm{ppm}$, which constituted the treatments in a randomized complete block design. It was found that by applying increasing doses of 6-benzylaminopurine, treatment 3 to $1.5 \mathrm{ppm}$ of 6-benzylaminopurine was the most satisfactory in terms of the number of outbreaks per explant. It is concluded that the BAP at $1.50 \mathrm{ppm}$ is the most optimal concentration for the propagation of T. tuberosum "mashua negra".
\end{abstract}

Keywords: Tropaeolum, mashua, in vitro, biotechnology, color pulp.

\footnotetext{
${ }^{\top}$ Universidad Nacional de Trujillo, Facultad de Ciencias Biológicas, Laboratorio de Biotecnología del Instituto de la Papa y Cultivos Andinos, Trujillo

"Autor de Correspondencia, e-mail: slopezm@unitru.edu.pe
} 


\section{INTRODUCCIÓN}

Tropaeolum tuberosum "mashua", es una planta herbácea de la familia Tropaeolaceae. Tiene su origen en Sudamérica y se cultiva entre 3000 a 4000 m.s.n.m., pudiéndose encontrar una gran diversidad de ecotipos. Su cultivo se remonta desde la época preinca, donde la "mashua" era usada en la alimentación humana de acuerdo a las actividades que desempeñaba y era considerada el tercer tubérculo después de oca y el olluco. Actualmente su cultivo se ha diseminado alrededor del mundo (Vásquez et al., 2010).

Las plantas de "mashua" presentan tallos de porte erecto y semi postrado, sus hojas son alternas y con peciolos cilíndricos de color verde oscuro en el haz y más claras en el envés. Poseen flores solitarias de distintos colores, además de raíces adventicias que les sirven de sostén, los tubérculos son tallos modificados de formas alargadas o fusiformes, el color de pulpa puede ser amarillo, blanco, rojizo, morado, gris y negro, dependiendo de la variedad (Aquise, 2011; Vásquez et al., 2010).

Los tubérculos de "mashua", son parte de la dieta diaria nutricional de los habitantes de menores recursos de la sierra central y sur del Perú. Poseen un alto contenido en proteínas, carbohidratos, fibras, carotenos y vitamina C. Dentro el punto de vista medicinal se ha demostrado la presencia de glucosinatos, los cuales tienen efectos beneficiosos en el sistema inmunológico, así como la presencia de polifenoles, sustancias químicas que evitan el desarrollo del cáncer por su acción antioxidante (Espin, 2013; Verástegui, 1993; Rivera, 2005; Cuya, 2009). Uno de los principales problemas de la mashua es su sabor poco agradable, motivo por el cual ha recibido menor atención por parte de la población urbana, catalogándola como "comida de pobres". Sin embargo, desde el punto de vista agronómico las ventajas de este cultivo es su rusticidad pudiendo cultivarse sin uso de fertilizantes, ni pesticidas (Surco, 2004; Verástegui, 1993).

La propagación in vitro se realiza con gran éxito ya que garantiza la producción de poblaciones de plántulas asépticas y de alta estabilidad genética (Villalobos y Thorpe, 1991). Para ello son necesarios la estandariza- ción de protocolos que faciliten su propagación masiva, como producto de la investigación en la concentración de los reguladores de crecimiento y de las sales minerales. Un fitorregulador muy empleado es la 6bencilaminopurina (BAP), esta citocinina se caracteriza por inhibir la dominancia apical, estimulando el brotamiento de las yemas laterales (Barceló et al., 1992; Gil y López, 2016; Márquez, 2018). Las consideraciones anteriormente expuestas nos permiten proponer como objetivo de la presente investigación, determinar la concentración más adecuada de la 6bencilaminopurina en la propagación in vitro de $T$. tuberosum "mashua" de pulpa de color.

\section{MATERIALY MÉTODOS}

La investigación se realizó en el Laboratorio de Biotecnología del Instituto de Papa y Cultivos Andinos de la Facultad de Ciencias Biológicas en la Universidad Nacional de Trujillo.

\section{Colecta de material biológico}

El material biológico consistente en tubérculos recién cosechados de T. tuberosum procedió de la Estación Experimental Carabamba del Instituto de la Papa y Cultivos Andinos. Una vez colectados, fueron transportados en bolsas de papel previamente rotulados al invernadero de la Facultad de Ciencias Biológicas.

\section{Instalación de experimento en invernadero}

En invernadero los tubérculos fueron sembrados en macetas, aplicándose 3 riegos semanales. Cuando la plántula alcanzo los 2 meses de edad, se colectaron entrenudos los cuales se transportaron a laboratorio (Figura 1).

\section{Preparación de medio MS (1962) y siembra de} explantes

Se utilizó el medio de cultivo constituido por el Medio Basal de Murashige y Skoog 1962, suplementados con sacarosa al 3\%, agar 0,8\% y dosis crecientes de 6bencilaminopurina, que constituyeron los tratamientos: $\mathrm{T} 1=0 ; \mathrm{T} 2=0,5 ; \mathrm{T} 3=1,5 \mathrm{yT} 4=2,5 \mathrm{ppm}$. luego se dispensó en tubos de ensayo para ser sellados con papel aluminio y esterilizados en autoclave a $120^{\circ} \mathrm{C}, 1$ atm de presión por 30 minutos (Figura 2). 


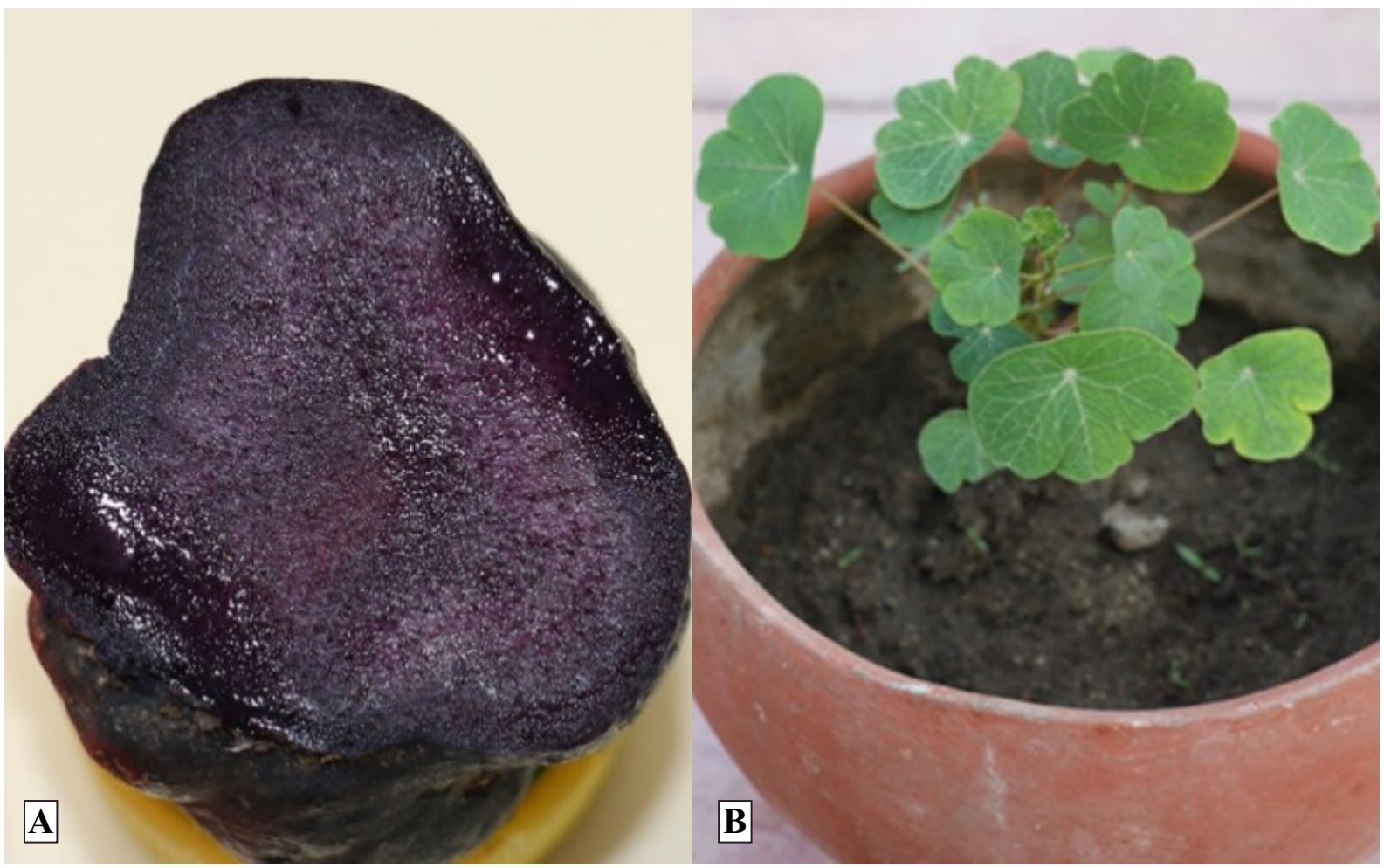

Figura 1. A, Tubérculos de T. tuberosum, mostrando el color de la cáscara y pulpa. B, Planta Madre donadora de explantes.

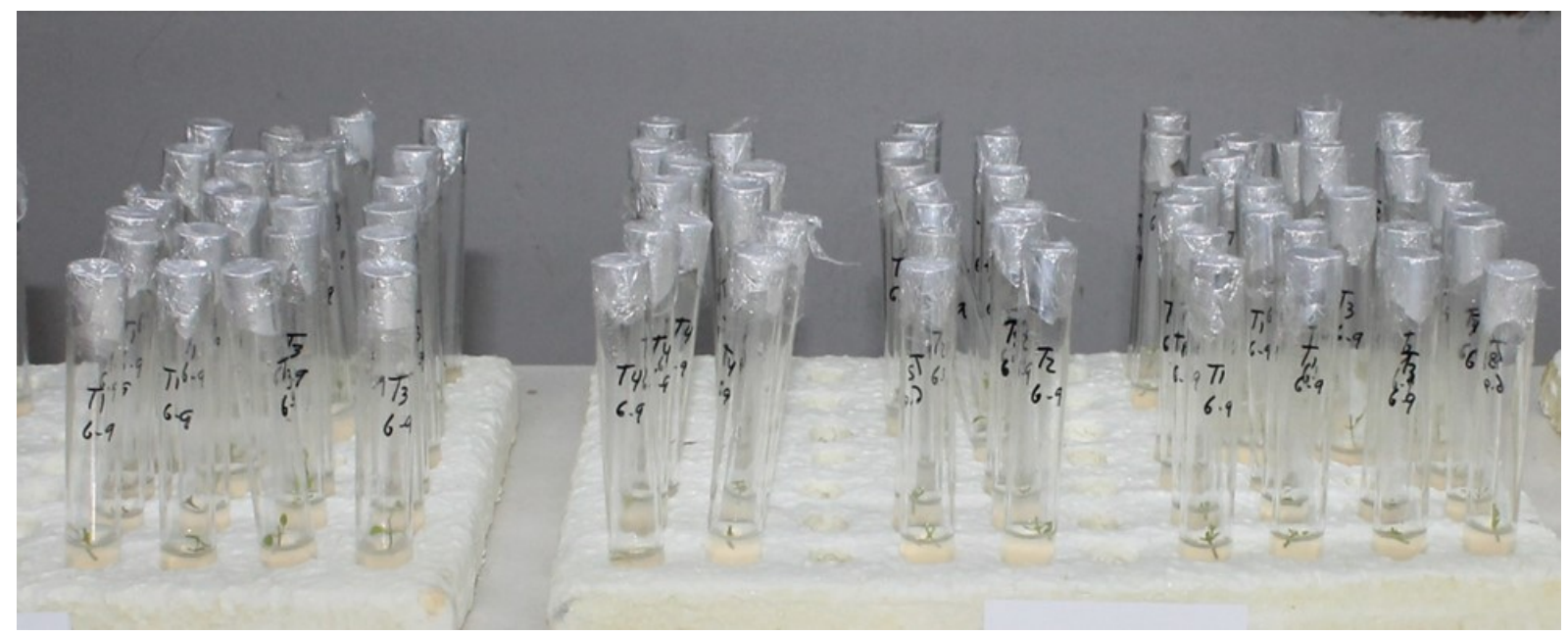

Figura 2. Diseño experimental con cuatro tratamientos, tres bloques y sies unidades muéstrales para la propagación in vitro de T. tuberosum"mashua".

\section{Siembra de nudos en medio sólido.}

En la cámara de flujo laminar, los explantes colectados de la planta madre fueron desinfectaron, primero con alcohol de $70^{\circ}$ durante 30 segundos, seguido de 4 enjuagues con agua estéril autoclavada, luego con lejía al 2\% durante 3 minutos, seguido de 4 enjuagues con agua estéril autoclavada. Se procedió a sembrar los nudos en sus respectivos medios de cultivo, para finalmente transportarlos al cuarto de incubación, donde se acondicionó luz fluorescente con un fotoperiodo de $16-8$ horas luz oscuridad, $18^{\circ} \mathrm{C}$ de temperatura y humedad relativa del 85\% (López et al., 2019).

\section{Análisis estadístico}

La evaluación se realizó a los 20 días del cultivo in vitro. Se empleó un diseño estadístico completamente aleatorizado. Los datos obtenidos, fueron sometidos a Análisis de Varianza y Test de diferencias significativas con probabilidad de error de 0,05 . 


\section{RESULTADOS}

Se encontró diferencias significativas entre tratamientos según el análisis de varianza (Tabla 1). Por otro lado, según el test de diferencias significativas de Tukey (Tabla 2), indica que el tratamiento T3 es el mejor para el número de brotes de T. tuberosum, obtenidos en condiciones in vitro.
Tabla 2. Prueba de Tukey para determinar las diferencias significativas entre los tratamientos $\mathrm{T} 1=0.00 ; \mathrm{T} 2=0,50$; $\mathrm{T} 3=1,50$ y $\mathrm{T} 4=2,50 \mathrm{ppm}$ sobre el número de brotes de $T$. tuberosum, obtenidos en condiciones in vitro

\begin{tabular}{cccc}
\hline Nivel & Casos & Media & Grupos Homogéneos \\
\hline 1 & 30 & 1,17 & $\mathrm{~A}$ \\
2 & 30 & 1,5 & $\mathrm{~B}$ \\
4 & 30 & 2,0 & $\mathrm{~B}$ \\
3 & 30 & 4,0 & $\mathrm{C}$ \\
\hline
\end{tabular}

Tabla 1. Análisis de Varianza (ANOVA) para determinar la influencia del 6-Bencilaminpopurina a concentraciones de $0.00 ; 0,50 ; 1,50$ y 2,50 ppm sobre el número de brotes de T. tuberosum, obtenidos en condiciones in vitro

\begin{tabular}{cccccc}
\hline Fuente & Suma de Cuadrados & g. l. & Cuadrado Medio & Razón-F & Valor-P \\
\hline Entre grupos & 144,8 & 3 & 48,2668 & 6998,68 \\
Intra grupos & 0,8 & 116 & 0,00689655 & \\
Total (Corr.) & 145,6 & 119 & & \\
\hline
\end{tabular}

\section{DISCUSIÓN}

La presencia de diferencias significativas entre tratamientos encontradas según el análisis de varianza (Tabla1), nos indica la acción de la 6-bencilaminopurina sobre la formación de brotes de T. tuberosum, es decir que a las concentraciones trabajadas el efecto es diferencial, ejerciéndose un efecto sinérgico favorable para la inducción de múltiples brotes (Gil y López, 2016). Otros protocolos sugieren el empleo de ANA a la concentración de 0.5-2 ppm y BAP a la concentración de 0.5-3 ppm, mientras que para la inducción a callos celulares se emplea 2, 4-D a la concentración de 5-10 ppm (Torres et al., 1989).

El comportamiento del tratamiento 3 (1,50 ppm), según test de diferencias significativas de Tukey, (Tabla 2), nos indica la acción inductora del 6bencilaminopurina en lograr inducir el mayor número de brotes por explante, probablemente se deba a que a 1,50 ppm el umbral de acción de esta citoquinina está en el pico más alto, diferenciándose en su acción a los otros tratamientos, lo cual nos permite tomar este dato como un estándar para la multiplicación masiva de esta especie en condiciones in vitro. Aspectos que concuerdan con las investigaciones de Márquez (2018), al emplear una concentración muy cercana en la propagación in vitro de T. tuberosum. Por otro lado, Carhuaz (2017), sostiene que la concentración de 2 ppm de 6- bencilaminopurina permite obtener múltiples brotes que al ser incubando en oscuridad completa se inducirá al proceso de tuberización in vitro.

\section{V.CONCLUSION}

Se concluye que el BAP a 1.50 ppm es la concentración más adecuada para la propagación in vitro de $T$. tuberosum "mashua negra" de pulpa de color.

\section{REFERENCIAS BIBLIOGRÁFICAS:}

Aquise, E. 2011. Cultivos andinos oca, mashua y ollu$c o$ 20011 . https://es.scribd.com/doc/108565205/ Cultivo-Andinos-Mashua-Oca-y-Olluco (Consultada el 5 de enero de 2020).

Barceló, J., G. Rodrigo, B. Sabater y R. Sánchez. 1992. Fisiología Vegetal 6ta. Edición. Madrid (España): Editorial Pirámide.

Carhuaz, R. K. 2017. Obtención de microtubérculos de Tropaeolum tuberosum Ruiz \& Pav. "mashua" mediante el sistema de inmersión temporal - Ayacucho, 2016. Tesis de Grado. Universidad Nacional de San Cristóbal de Huamanga. Ayacucho (Perú).

Cuya, R. 2009. Efecto de secado en bandeja y atomización sobre la actividad antioxidante de la mashua (Tropaeolum tuberosum). Tesis de Maestría. Universidad Nacional Agraria La Molina. Lima (Perú).

Espin, C. 2013. Aporte al rescate de la mashua aplicando técnicas de cocina de vanguardia. Tesis de Grado. Universidad de Cuenca. Cuenca(Ecuador).

Gil, E. y E. López. 2016. "Efecto sinérgico del ácido 
indolacético, ácido giberélico y 6bencilaminopurina en la propagación in vitro de "papaya” Carica papaya L. (Caricaceae).” Arnaldoa 23 (2): 577-586.

Márquez, C. 2018. Desarrollo de un protocolo de introducción y multiplicación rápida de Tropaeolum tuberosum (mashua negra), empleando la propagación in vitro. Tesis pregrado. Universidad Católica de Santa María. Arequipa (Perú).

Lopéz, S., J. Mostacero, E. Gil, A. Lopéz., y J. De la Cruz. 2019. "Efecto del ácido giberélico y del ácido indolacético en la micropropagación in vitro de Solanum tuberosum var. Maria Reiche." Rebiol39 (1): 1-9.

Rivera, F. 2005. Raices y tubérculos con alto contenido energético y medicinal 2005. http://repositorio.educacionsuperior.gob.ec/h andle/28000/675 (Consultada el 5 de enero de 2020).

Surco, F. 2004. Caracterización de almidones aislados de tubérculos andinos: mashua (Tropaeolum tuberosum), oca (Oxalis tuberosa), olluco (Ullucus tuberosus) para su aplicación tecnológica. Tesis de Maestria. Universidad Nacional Mayor de San Marcos. Lima (Perú).

Torres O., T. Fandiño, y M. Perea. 1989. “Aspectos anatómicos y fisiológicos de cultivos in vitro de Tropaeolum tuberosum (Ruiz \& Pavón)." Acta Biológica Colombiana. 1(5): 71-79.

Vásquez L., G. Vásquez, J. Escurra, y R. Aguirre. 2010. Plantas Medicinales del Norte de Perú. Lambayeque: Perú: FINCYT y UNPRG.

Verástegui, Y. 1993. Inducción in vitro de tubérculos Tropaeolum tuberosum Ruiz y Pavón "Mashua”. Tesis de Grado. Universidad Nacional de Trujillo. Trujillo (Perú).

Villalobos, V. y T. Thorpe. 1991. Micropropagación: Conceptos, metodología y resultados. En: Cultivo de Tejidos en la agricultura. Fundamentos y Aplicaciones. http://exa.unne.edu.ar/biologia/fisiologia.veg
etal/Cultivo\%20de\%20Tejidos\%20en\%20la \%20Agricultura/capitulo6.pdf (Consultada el 5 de enero de 2020). 